\title{
Effects of corticosterone pellets on baseline and stress-induced corticosterone and corticosteroid-binding-globulin
}

\author{
Claudia Müller ${ }^{\mathrm{a}, \mathrm{b}, *}$, Bettina Almasi ${ }^{\mathrm{a}, \mathrm{b}}$, Alexandre Roulin ${ }^{\mathrm{c}}$, Creagh W. Breuner ${ }^{\mathrm{d}}$, \\ Susanne Jenni-Eiermann ${ }^{a}$, Lukas Jenni ${ }^{\text {a }}$ \\ a Swiss Ornithological Institute, Luzernerstrasse 6, CH-6204 Sempach, Switzerland \\ ${ }^{\mathrm{b}}$ Zoological Museum of the University of Zurich, Winterthurerstrasse 190, CH-8057 Zurich, Switzerland \\ ${ }^{\mathrm{c}}$ Department of Ecology and Evolution, University of Lausanne, UNIL Sorge, Le Biophore, CH-1015 Lausanne, Switzerland \\ ${ }^{\mathrm{d}}$ Division of Biological Sciences, University of Montana, 32 Campus Drive, Missoula, MT 59812, USA
}

\section{A R T I C L E I N F O}

Article history:

Received 20 August 2008

Revised 2 October 2008

Accepted 15 October 2008

Available online 1 November 2008

\section{Keywords:}

Falco tinnunculus

Tyto alba

Corticosterone administration

Self-degrading implants

Corticosteroid-binding-globulin

Acute stress response

Negative feedback regulation

Aves

Post-natal

\begin{abstract}
A B S T R A C T
Exogenous administration of glucocorticoids is a widely used and efficient tool to investigate the effects of elevated concentrations of these hormones in field studies. Because the effects of corticosterone are dose and duration-dependent, the exact course of plasma corticosterone levels after exogenous administration needs to be known. We tested the performance of self-degradable corticosterone pellets (implanted under the skin) in elevating plasma corticosterone levels. We monitored baseline (sampled within 3 min after capture) total corticosterone levels and investigated potential interactions with corticosteroid-binding-globulin (CBG) capacity and the endogenous corticosterone response to handling in Eurasian kestrel Falco tinnunculus and barn owl Tyto alba nestlings. Corticosterone pellets designed for a 7-day-release in rodents elevated circulating baseline total corticosterone during only 2-3 days compared to placebo-nestlings. Highest levels occurred 1-2 days after implantation and levels decreased strongly thereafter. CBG capacity was also increased, resulting in a smaller, but still significant, increase in baseline free corticosterone levels. The release of endogenous corticosterone as a response to handling was strong in placebo-nestlings, but absent 2 and 8 days after corticosterone pellet implantation. This indicates a potential shut-down of the hypothalamo-pituitary-adrenal axis after the 2-3 days of elevated baseline corticosterone levels. 20 days after pellet implantation, the endogenous corticosterone response to handling of nestlings implanted with corticosterone pellets attained similar levels as in placebo-nestlings. Self-degradable pellets proved to be an efficient tool to artificially elevate circulating baseline corticosterone especially in field studies, requiring only one intervention. The resulting peak-like elevation of circulating corticosterone, the concomitant elevation of CBG capacity, and the absence of an endogenous corticosterone response to an acute stressor have to be taken into account.
\end{abstract}

(c) 2008 Elsevier Inc. All rights reserved.

\section{Introduction}

Exogenous administration of glucocorticoids is a widely used and efficient tool to investigate the effects of elevated concentrations of these hormones. Methods to artificially elevate glucocorticoids include single or repeated injections (e.g. Remage-Healey and Romero, 2002; Loiseau et al., 2008), adding hormone to the food or drinking water (e.g. Breuner et al., 1998; Hiebert et al., 2000; Hull et al., 2007), or implantation of a silastic tube filled with crystalline hormone (e.g. Silverin, 1986; Wingfield and Silverin, 1986; Kitay-

\footnotetext{
* Corresponding author. Swiss Ornithological Institute, Luzernerstrasse 6, CH6204 Sempach, Switzerland. Fax: +41 414629710

E-mail address: claudia.mueller@vogelwarte.ch (C. Müller)
}

sky et al., 2001), a gelling material containing hormone (French et al., 2007), a mini-infusion pump (Donker and Beuving, 1989) or an osmotic pump (Horton et al., 2007). These various forms of administration have different advantages and disadvantages. Injections imply repeated handling with concurrent internal adrenocortical responses when hormone levels should be elevated over days. While the intake of glucocorticoids with food or water is not invasive, the amount of assimilated glucocorticoids depends on the quantity of food or water ingested and can vary between individuals. Additionally, repeated injections and hormone consumption are very difficult to apply in free-living animals. Glucocorticoidreleasing implants or osmotic pumps require only one intervention followed by the release of a given amount of glucocorticoids over a longer period of time and are often the method of choice in field studies. However, removal of these implants requires a second intervention. An alternative method is the implantation of self- 
degradable corticosterone-releasing pellets, which should provide a constant release by biodegradation of the matrix and do not require subsequent removal. This method has rarely been used in experiments with wild bird species until now (e.g. Pravosudov, 2003; Bourgeon and Raclot, 2006; Bonier et al., 2007).

In most studies the effect of corticosterone administration on circulating corticosterone is not well documented, although the levels of circulating corticosterone attained and the duration of the elevation are supposed to be decisive, since the effects of corticosterone are dose dependent (e.g. Romero, 2004) and the duration of the exposure to elevated levels is crucial. The time course of circulating baseline (sampled within 3 min after capture) corticosterone after administering corticosterone in birds has been followed in only a few studies by measuring circulating total corticosterone at a few specific time points, e.g. after several days (e.g. Kitaysky et al., 2001). The interactions further up and downstream from corticosterone during and after an exogenous corticosterone administration have been investigated predominantly in mammals (e.g. Feldman et al., 1979; Zhao et al., 1997) and only rarely in birds. Only one study investigated the effect of corticosterone administration on corticosteroid-binding-globulin (CBG) (Breuner et al., 2003b). We know of no study examining the response to an acute stressor after the period when circulating corticosterone was elevated.

The aim of this paper is to report our experiences with selfdegradable corticosterone-releasing pellets in an extended field study with nestling Eurasian kestrels Falco tinnunculus and barn owls Tyto alba. Specifically, we investigated (a) the time course of total circulating corticosterone during 20 days after implantation; (b) whether CBG capacity and estimated free corticosterone levels were affected by corticosterone administration, and (c) whether the response of circulating corticosterone levels to an acute stressor (handling) was affected by corticosterone administration. We predicted that CBG capacity would increase with elevated corticosterone levels and that the negative feedback regulation would decrease the endogenous response to an acute stressor in cortnestlings with elevated corticosterone compared to placebo-nestlings. For aim (b) and (c) we were interested in the acute effects of the implant and in potential mid-term effects after circulating baseline corticosterone had returned to normal levels.

\section{Material and methods}

\subsection{Study species and study sites}

The Eurasian Kestrel is a small, diurnal raptor. Usually, the female starts incubation after laying the third of 4-6 eggs, hence, the three oldest nestlings have about the same age. The nestlings stay in the nest for 32-39 days and reach their maximal body mass around day 23. Fieldwork was performed in North-Western Switzerland $\left(47^{\circ} 25^{\prime} \mathrm{N} / 7^{\circ} 50^{\prime} \mathrm{E}\right)$, where kestrels raise their young in nest boxes mounted on agricultural buildings in open rural landscapes. Mean brood size ( \pm SD) of the investigated broods at the day of implantation was $4.4 \pm 0.96$ nestlings, mean hatching date was on 7 June \pm 13 days.

The barn owl is a medium-sized, nocturnal owl species producing clutches of 2-11 eggs. Incubation starts after laying the first egg and only females incubate. The laying intervals of two to three days entail a pronounced within-brood age hierarchy. The nestlings reach their maximum body mass with 40 days and fledge with about 56 days of age. Barn owls were investigated in Western Switzerland $\left(46^{\circ} 49^{\prime} \mathrm{N} / 06^{\circ} 56^{\prime} \mathrm{E}\right)$, where they breed in nest boxes attached on barns and farm buildings. Mean brood size $( \pm S D)$ of the investigated broods at the day of implantation was $5.8 \pm 1.54$ nestlings, mean hatching date was on 3 June \pm 33 days.

\subsection{Experimental corticosterone treatment}

The experiment was carried out in 109 kestrel nestlings of 30 broods (13 in 2004 and 17 in 2005) and 208 barn owl nestlings of 73 broods (33 in 2004, 19 in 2005, 21 in 2006). Hatching date was determined through regular nest box controls.

Two randomly selected nestlings out of the four oldest within a brood were implanted with a self-degradable corticosteronereleasing pellet on nestling day 13 (mean age \pm SD: $13.2 \pm 1.4$ days) in the kestrel and nestling day 25 (mean age $27 \pm 5$ days) in barn owl nestlings. Pellets designed for a 7-day-release in rodents were obtained from Innovative Research of America (Sarasota, FL, USA.). Because kestrels were lighter in body mass (mean \pm SD: $165 \pm 22 \mathrm{~g}$ at implantation) than barn owls (294 $\pm 69 \mathrm{~g}$ at implantation), we implanted a $10 \mathrm{mg}$ corticosterone pellet (cat \# C-111) in nestling kestrels and a $15 \mathrm{mg}$ corticosterone pellet (cat \# G-111) in nestling barn owls. The other two of the four oldest siblings were implanted with a corresponding placebo pellet, creating two treatment groups (cort- and placebo-nestlings). Before implantation, body mass (kestrels: $t=1.44, \mathrm{df}=107, p=0.153$; barn owls: $t=0.71$, $\mathrm{df}=206, \mathrm{p}=0.478$ ), nestling age (kestrels: $t=0.56, \mathrm{df}=107$, $p=0.577$; barn owls: $t=0.28, \mathrm{df}=206, p=0.783$ ) and the proportion of the sexes (kestrels: $\mathrm{X}^{2}=0.01, \mathrm{df}=1, p=0.922$; barn owls: $\mathrm{X}^{2}=0.09, \mathrm{df}=1, p=0.752$ ) did not differ between cort- and placebo-nestlings. The pellets were placed under the skin of the flank above the knee through a small incision. The pellets are very sensitive to alcoholic solvents, even when seemingly evaporated (based on our own observations in previous trials, and personal communication by the provider). Therefore, to prevent accelerated corticosterone release, the skin was not disinfected. The incision was closed with tissue adhesive (Histoacryl ${ }^{\circledR}$, Braun, Germany). If more than four siblings were present, the fifth and following were not implanted. All methods described in this study were approved by the Swiss committee for animal research (animal experiment permit no 274 from the Cantonal Veterinarian Office of Baselland for kestrels and no 1736 from the Veterinarian Office of Vaud for barn owls).

Pellet implantation and corticosterone treatment did not affect survival of nestlings. In kestrels nestlings, between nestling day 13 and fledging, 54 of 56 placebo-nestlings (96.4\%) and 60 of 61 cortnestlings (98.4\%) survived, while 242 of 260 nestlings not treated with a pellet survived (93.1\%; nestlings of other broods at our study site during the study years which were only measured and blood sampled). In barn owls, 40 of 43 cort-nestlings (93.0\%) and 42 of 43 placebo-nestlings (97.7\%) survived until fledging, while 159 of 179 non-treated nestlings (88.8\%) survived until fledging. All differences in survival between cort-implanted, placebo-implanted and non-implanted birds were not significant (kestrel: Pearson $\mathrm{X}^{2}=3.12$, $\mathrm{df}=2, p=0.210$; barn owl: Pearson $\mathrm{X}^{2}=3.57$, $\mathrm{df}=2, p=0.168)$.

\subsection{Blood sampling}

We took baseline blood samples in kestrel nestlings 3 days before implantation, on the day of implantation (day 0 ) and 3 and 8 days after implantation. In a subgroup of kestrel nestlings we took an additional baseline sample 1 or 2 days after implantation. All baseline samples were taken within 3 min after taking nestlings out of the nest box in batches of two. There was no effect of batch number or the time between first arrival and bleeding on baseline corticosterone $(F=0.57, \mathrm{df}=2, p=0.567)$. In barn owls we collected a blood sample within 3 min after having opened the nest box on the day of implantation and 6 and 20 days after implantation (blood samples taken after $3 \mathrm{~min}$ were not used for this study); a subgroup was also sampled 2 or 3 days after implantation. In addition, we had baseline samples of 5 cort-nestlings of 4 
broods 1 day after implantation which we used to demonstrate whether corticosterone levels were not pharmacologically high, but we did not include them in the statistical tests, because the corresponding placebo samples were missing. After taking the first blood sample, the nestlings were measured, weighed and then held in a cloth bag. The adrenocortical response to handling was assessed by taking a second blood sample about 17 min (mean \pm SD: $16.9 \pm 1.4 \mathrm{~min}$, range: $15-22$ ) after taking the nestlings out of the nest box in kestrels and $19 \mathrm{~min}$ (19.13. $\pm 2.92 \mathrm{~min}$, range: 15-25) in barn owls. Handling-induced corticosterone levels did not vary within these time ranges (Placebo-nestlings: $p>0.242$ ). Blood was sampled by puncturing the alar vein and collected in heparinized capillary tubes. Within $30 \mathrm{~min}$, the blood was centrifuged in Eppendorf tubes and the plasma immediately stored in liquid nitrogen in the field and at $-20^{\circ} \mathrm{C}$ once in the laboratory.

\subsection{Corticosterone assay}

Plasma corticosterone concentration was determined using an enzyme immuno assay (Munro and Stabenfeldt, 1984; Munro and Lasley, 1988). Five microliter of plasma was added to $195 \mu \mathrm{l}$ water, and from this solution we extracted corticosterone with $4 \mathrm{ml}$ dichloromethane, which was re-dissolved in phosphate buffer and measured in triplicates in the enzyme immunoassay. The dilution of the corticosterone antibody (Chemicon; cross-reactivity: 11-dehydrocorticosterone $0.35 \%$, Progesterone $0.004 \%, 18-\mathrm{OH}-$ DOC $0.01 \%$, Cortisol 0.12\%, 18-OH-B 0.02\% and Aldosterone 0.06\%) was 1:8'000. HRP (1:400'000) linked to corticosterone served as enzyme label and ABTS as substrate. The concentration of corticosterone in plasma samples was calculated by using a standard curve run in duplicate on each plate. Plasma pools from chickens with two different corticosterone concentrations were included as internal controls on each plate. In 38 cases the concentration was below the detection threshold, and in this case the determination was repeated with $10 \mu$ plasma. If the concentration was still below the detection threshold (27 samples), the value of the lowest detectable concentration ( $1 \mathrm{ng} \mathrm{ml}^{-1}$ ) was assigned. Intra-assay variation ranged from $4.5 \%$ to $13.4 \%$ and inter-assay variation from $9.6 \%$ to $23.0 \%$, depending on the concentration of the internal control and the year of determination.

\subsection{Corticosteroid-binding-globulin}

The affinity and capacity of corticosteroid-binding-globulin (CBG) was measured with a radioligand-binding assay with tritiated corticosterone following Breuner et al. (2003a). For point sample analysis, plasma (10-15 $\mu$ l in the kestrel, $5 \mu \mathrm{l}$ in the barn owl) was stripped of endogenous steroids with two parts of dextrancoated charcoal ( $0.1 \%$ dextran, $1 \%$ Norit A charcoal in $50 \mathrm{mM}$ Tris) for $30 \mathrm{~min}$ at room temperature. Outside this stripping procedure, the plasma was maintained below $4{ }^{\circ} \mathrm{C}$. The final assay dilution of kestrel plasma samples was 1:99, those of the barn owl samples $1: 450$. The binding assay was carried out in $50 \mathrm{nM}$ Tris buffer at $4{ }^{\circ} \mathrm{C}$ and terminated after $2 \mathrm{~h} .1 \mathrm{~h}$ before filtering, glass fiber filters (Whatman) were soaked in $25 \mathrm{nM}$ Tris with $0.3 \%$ polyethyleneimine. After filtration, filters were rapidly rinsed with three rinses of $3 \mathrm{ml}$ ice-cold $25 \mathrm{nM}$ Tris. Point sample analysis was performed with individual plasma samples, for the saturation analyzes pooled samples were run. For the saturation analyzes, $0.25-12 \mathrm{nM}\left[{ }^{3} \mathrm{H}\right]$ Corticosterone were incubated with pooled plasma of each species with and without $1 \mu \mathrm{M}$ unlabeled corticosterone. $20 \mathrm{nM}\left[{ }^{3} \mathrm{H}\right]$ Corticosterone was employed to estimate CBG capacity in individual birds. Affinity estimates obtained from equilibrium saturation analysis ( $\mathrm{Kd}=4.59$ and $4.11 \mathrm{nM}$ in kestrels and barn owls, respectively, Fig. $1 \mathrm{~A}$ and $\mathrm{B}$ ) indicated that this ligand concentration occupies $\sim 80 \%$ of total binding sites. For analysis, the samples were
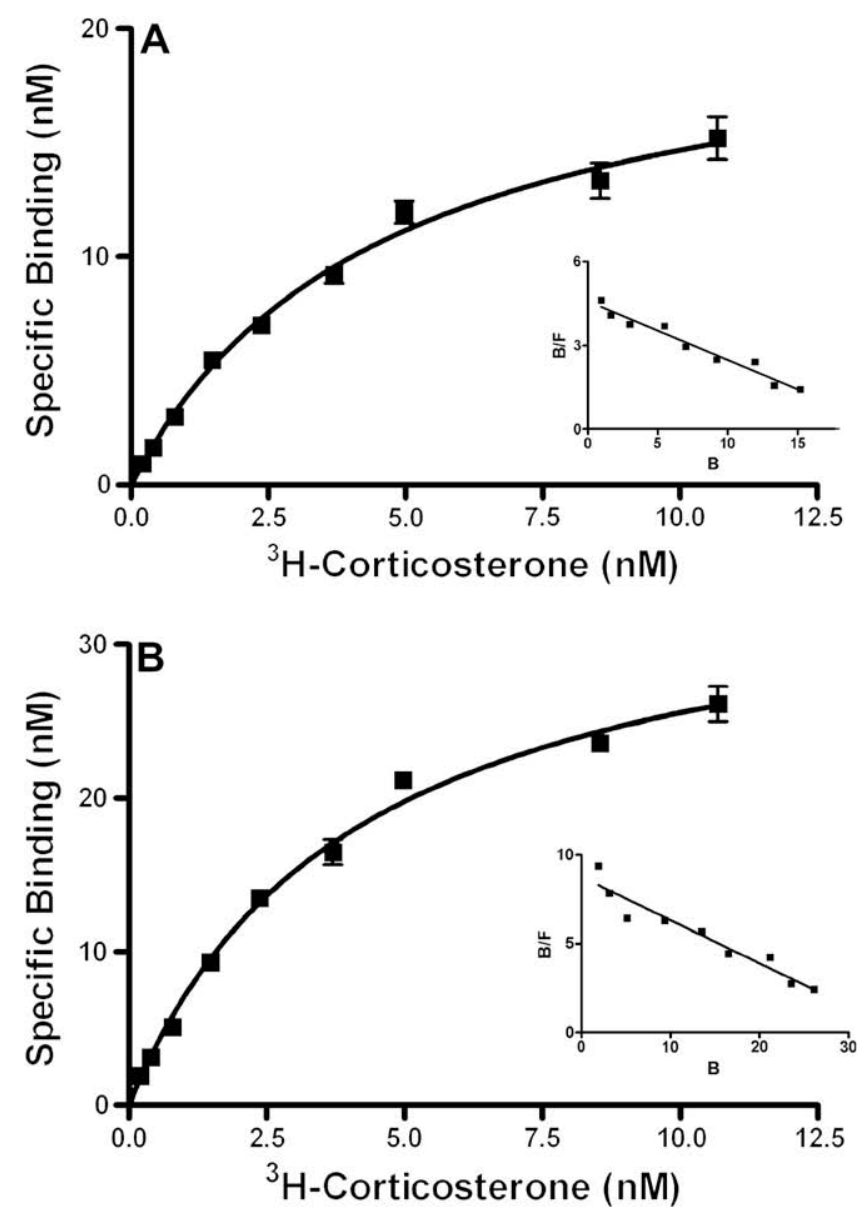

Fig. 1. Equilibrium saturation binding curve demonstrating specific binding of ${ }^{3} \mathrm{H}$ corticosterone to (A) Eurasian kestrel and (B) barn owl plasma as a function of increasing concentrations of radiolabeled corticosterone. Points indicate means \pm SE. The inlay is the Scatchard-Rosenthal replot of the data.

corrected to $100 \%$ capacity within each assay. The intra-assay variation was $5.7 \%$ and $7.1 \%$, the inter-assay variation $7.58 \%$ and $17.1 \%$ (kestrel and barn owl samples determined separately).

The equation of Barsano and Baumann (1989) was used to estimate free corticosterone titers from total corticosterone concentrations and $\mathrm{CBG}$ binding parameters

$$
\begin{aligned}
H_{\text {free }}= & 0.5 \times\left[H_{\text {total }}-B_{\max }-1 / K_{\mathrm{a}}\right] \\
& \pm \sqrt{\left(B_{\max }-H_{\text {total }}+1 / K_{\mathrm{a}}\right)^{2}+4\left(H_{\text {total }} / K_{\mathrm{a}}\right)}
\end{aligned}
$$

where $H_{\text {free }}$ is free Hormone, $H_{\text {total }}$ is total Hormone, $B_{\max }$ is total binding capacity of $\mathrm{CBG}$, and $K_{\mathrm{a}}=1 /$ dissociation constant $\left(K_{\mathrm{d}}\right)$ (all values in $\mathrm{nM}$ ). Corticosterone was analyzed in all baseline and handling induced blood samples, CBG capacity was measured in all baseline kestrel and barn owl samples and a subsample of the handling induced kestrel samples (a subsample of day 10, all samples of day 21).

\subsection{Statistical analyses}

To analyze the overall effect of corticosterone treatment on circulating corticosterone we performed mixed models (REML, Genstat 10 ) separately with total corticosterone, CBG capacity and free corticosterone as dependent variables. We included treatment (corticosterone versus placebo) and time after implantation (number of days after implantation) and their interaction as fixed factors and nestling identity nested in broods as random factors to correct for the dependence of siblings within a brood. 
The effect of corticosterone treatment on the response to handling was analyzed separately for total and free corticosterone and CBG capacity by running a repeated measures analysis including baseline and stress-induced levels of those days with two blood samples per individual. The model included time after implantation, treatment, blood sample (baseline versus handling-induced) and their interactions as fixed factors, and nestling identity nested in brood as random factors. To compare corticosterone levels of baseline and handling-induced blood samples and CBG capacity between treatment groups at the different sampling days, we performed post-hoc tests for each day separately. Treatment was included as fixed and brood as random factor in these mixed models and significance levels were adjusted according to Bonferroni (Sokal and Rohlf, 2000). Kestrel and barn owl data were analyzed in separate models.

\section{Results}

\subsection{Effect of corticosterone pellets on baseline total and free corticosterone levels and CBG capacity}

Implanting a corticosterone pellet had a highly significant effect on the plasma concentration of total corticosterone in both species (Table 1 and 2). Before implantation, there was no difference in total corticosterone level between cort- and placebo-nestlings in both species (Fig. 2A and B). Total corticosterone in kestrel cortnestlings was significantly elevated over placebo-nestlings 1 day after implantation (post-hoc test: $F=39.14, \mathrm{df}=1,10.4, p<0.001$, Fig. 2A, Fig. 3A, range: $29.78-63.80 \mathrm{ng} / \mathrm{ml}$ ), and tended towards significance 2 days after implantation $(F=7.92, \mathrm{df}=1,4.6$, $p=0.037$, not significant at the Bonferroni-corrected $p$-level of 0.013); corticosterone levels were indistinguishable from those of placebo-nestlings 3 and 8 days after implantation. In barn owl nestlings, total corticosterone in cort-nestlings was significantly increased 2 days after implantation $(F=66.78, \mathrm{df}=1,80.0$, $p<0.001$, Fig. 2B, Fig. 3B, range 2 days after implantation: $8.11-$ $118.03 \mathrm{ng} / \mathrm{ml}$ ) and returned to the level of placebo-nestlings 3, 6 and 20 days after implantation $(p>0.161)$. The five values of cort-nestlings 1 day after implantation (range $10.7-48.1 \mathrm{ng} / \mathrm{ml}$ ) indicated that levels were not higher than 2 days after implantation.

CBG capacity in kestrel nestlings did not significantly vary with time or treatment (Table 1 ), and CBG capacity was not significantly elevated on day 1 and 2 after cort-implantation (Fig. 2C; 1 day: $F=0.80, \quad \mathrm{df}=1,13.0, \quad p=0.386$, day $2: F=1.58, \mathrm{df}=1,2.3$, $p=0.335$ ). In barn owl nestlings, there was an overall effect of treatment and time and their interaction on CBG capacity (Table 2, Fig. 2D). CBG capacity of barn owl cort-nestlings was significantly elevated 2 days after implantation $(F=37.13$, df $=1,69.7$, $p<0.001$ ) and indistinguishable from placebo-nestlings 3, 6 and 20 days after implantation.

Estimated free corticosterone levels varied with time and treatment group depending on time after implantation in both species
(Table 1 and 2). There was no difference between the treatment groups before implantation in both species (Fig. $2 \mathrm{E}$ and $\mathrm{F}$ ). In kestrels, although the interaction term time after implantation $\mathrm{x}$ treatment was highly significant (Table 1), the Bonferroni-corrected post-hoc tests indicated just no significant difference between cort-nestlings and placebo-nestlings one day after implantation $(F=5.33, \mathrm{df}=1,13.0, p=0.038$, Fig. 2E, not significant at the Bonferroni-corrected $p$-level of 0.013 ), and no difference between the treatment groups 2,3 and 8 days after implantation $(p=0.236$, 0.847 and 0.072 , respectively). In barn owl nestlings, free corticosterone of cort-nestlings was significantly elevated two days after implantation, $(F=26.06$, df $=77.8, p<0.001)$, while 3,6 and 20 days after implantation there was no difference between the treatment groups ( $p=0.657,0.153$ and 0.733 , respectively, Fig. $2 F$ ).

\subsection{Effect of corticosterone pellets on handling-induced total and free corticosterone levels and CBG capacity}

In both species, total and free corticosterone levels depended on time after implantation, treatment group and blood sample (baseline versus handling-induced) (interaction time $\times$ treatment group $\times$ blood sample $p<0.001$, Table 3 ).

Placebo-nestlings of both species showed a marked increase of total plasma corticosterone levels as a response to handling. In cort-nestlings 2 days after implantation (barn owl, Fig. 4B), when baseline total corticosterone levels were elevated, plasma corticosterone levels increased only little as a response to handling and reached similar levels as in placebo-nestlings (treatment: $F=2.33$, $\mathrm{df}=1,120.8, p=0.130$ ). In cort-nestlings 8 days after implantation (kestrel, Fig. 4A), when baseline levels were low again, the adrenocortical response to handling was virtually absent (interaction time after implantation $\times$ treatment group $\times$ blood sample $p<0.001$, Table 3, Fig. 4A). In barn owl nestlings 20 days after implantation, when baseline levels were similar in both treatment groups, cort-nestlings reached similar handling-induced levels as the placebo-nestlings $(F=3.99, \mathrm{df}=1,37.6, p=0.053$, Fig. 4B).

\section{Discussion}

\subsection{Effect of corticosterone pellets on total baseline corticosterone levels}

The self-degradable corticosterone pellets clearly increased circulating total baseline corticosterone levels in kestrel and barn owl nestlings 1-3 days after implantation compared with placebo-nestlings. With the $10 \mathrm{mg}$ and $15 \mathrm{mg}$ 7-day-release pellets used in this study, the increase in corticosterone levels was within the range reached by handling before the treatment (kestrel nestlings 8$50 \mathrm{ng} / \mathrm{ml}$ after $17 \mathrm{~min}$; barn owl nestlings $10-110 \mathrm{ng} / \mathrm{ml}$ after $19 \mathrm{~min}$ ) and therefore, within the physiological range. Although the increase in corticosterone levels induced by the pellets varied between individuals, they were clearly higher than in placebo-nes-

Table 1

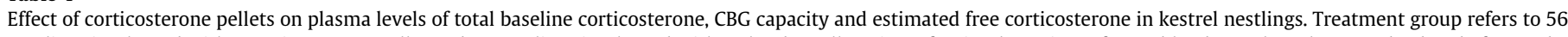

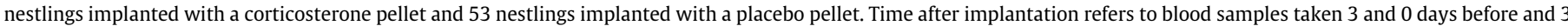

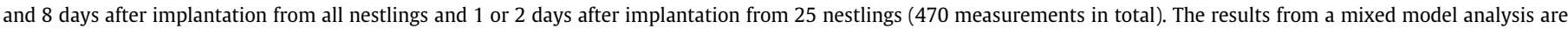
given with nestling identity nested in broods as random factors.

\begin{tabular}{|c|c|c|c|c|c|c|c|c|c|}
\hline & \multicolumn{3}{|c|}{ Total corticosterone } & \multicolumn{3}{|c|}{ CBG capacity } & \multicolumn{3}{|c|}{ Free corticosterone } \\
\hline & df & F statistic & $\mathrm{F} \mathrm{pr}$ & df & F statistic & $\mathrm{F} \mathrm{pr}$ & df & F statistic & $\mathrm{F} \mathrm{pr}$ \\
\hline Time after implantation & $5,438.8$ & 29.83 & $<0.001$ & $5,308.2$ & 1.44 & 0.210 & $5,320.6$ & 14.37 & $<0.001$ \\
\hline Treatment group & $1,433.5$ & 7.34 & 0.007 & $1,73.7$ & 0.02 & 0.896 & $1,78.2$ & 1.26 & 0.265 \\
\hline Time after implantation $\times$ Treatment group & $5,429.7$ & 33.04 & $<0.001$ & $5,314.5$ & 2.05 & 0.071 & $5,323.5$ & 15.78 & $<0.001$ \\
\hline
\end{tabular}


Table 2

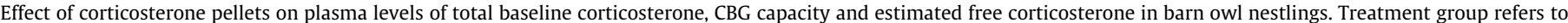

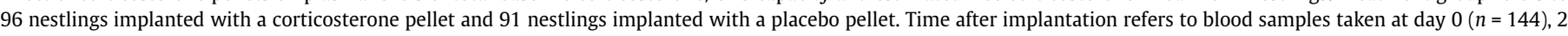

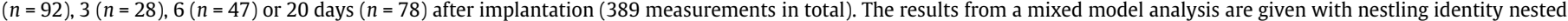
in broods as random factors.

\begin{tabular}{|c|c|c|c|c|c|c|c|c|c|}
\hline & \multicolumn{3}{|c|}{ Total corticosterone } & \multicolumn{3}{|c|}{ CBG capacity } & \multicolumn{3}{|c|}{ Free corticosterone } \\
\hline & df & F statistic & $\mathrm{F} \mathrm{pr}$ & df & F statistic & $\mathrm{F} \mathrm{pr}$ & df & F statistic & $\mathrm{F} \mathrm{pr}$ \\
\hline Time after implantation & $4,362.3$ & 28.50 & $<0.001$ & $4,270.1$ & 10.52 & $<0.001$ & $4,355.2$ & 7.99 & $<0.001$ \\
\hline Treatment group & $1,367.0$ & 46.47 & $<0.001$ & $1,113.7$ & 18.59 & $<0.001$ & $1,339.4$ & 9.19 & 0.003 \\
\hline Time after implantation $\times$ Treatment group & $4,345.8$ & 28.39 & $<0.001$ & $4,288.8$ & 10.15 & $<0.001$ & $4,325.5$ & 8.10 & $<0.001$ \\
\hline
\end{tabular}

Kestrel
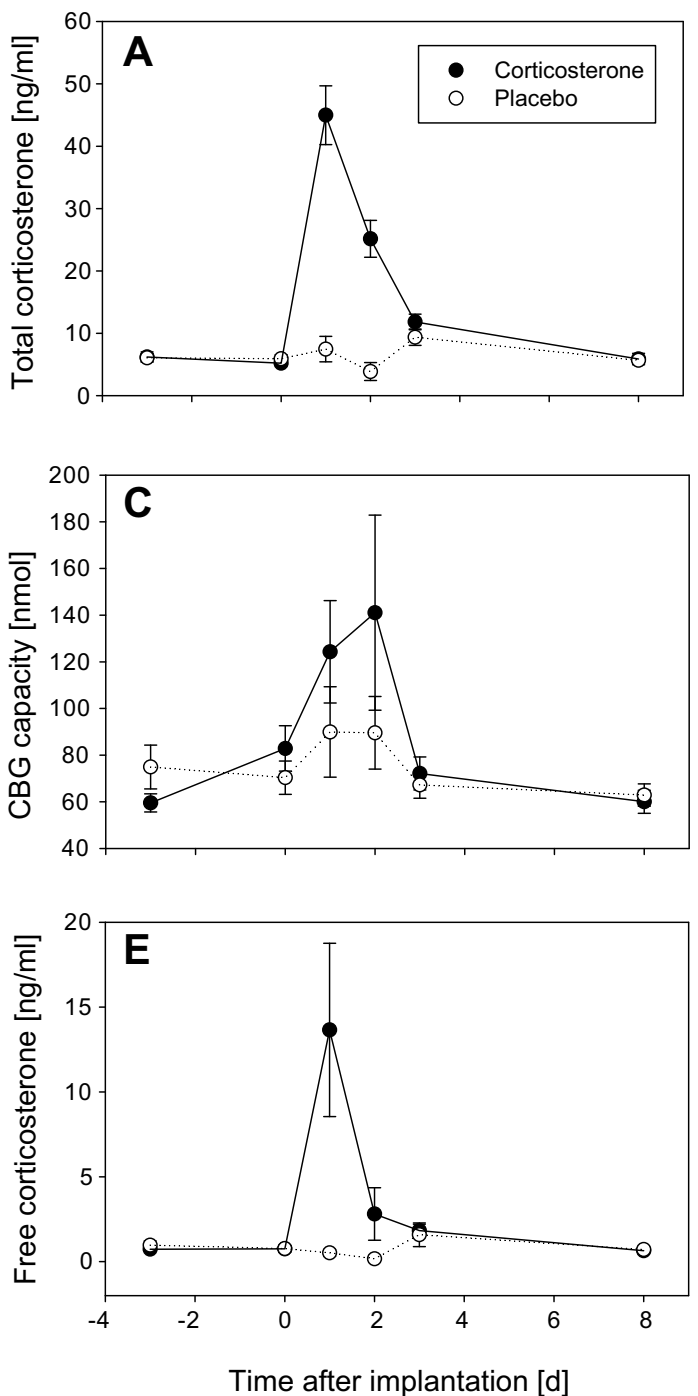

Barn owl
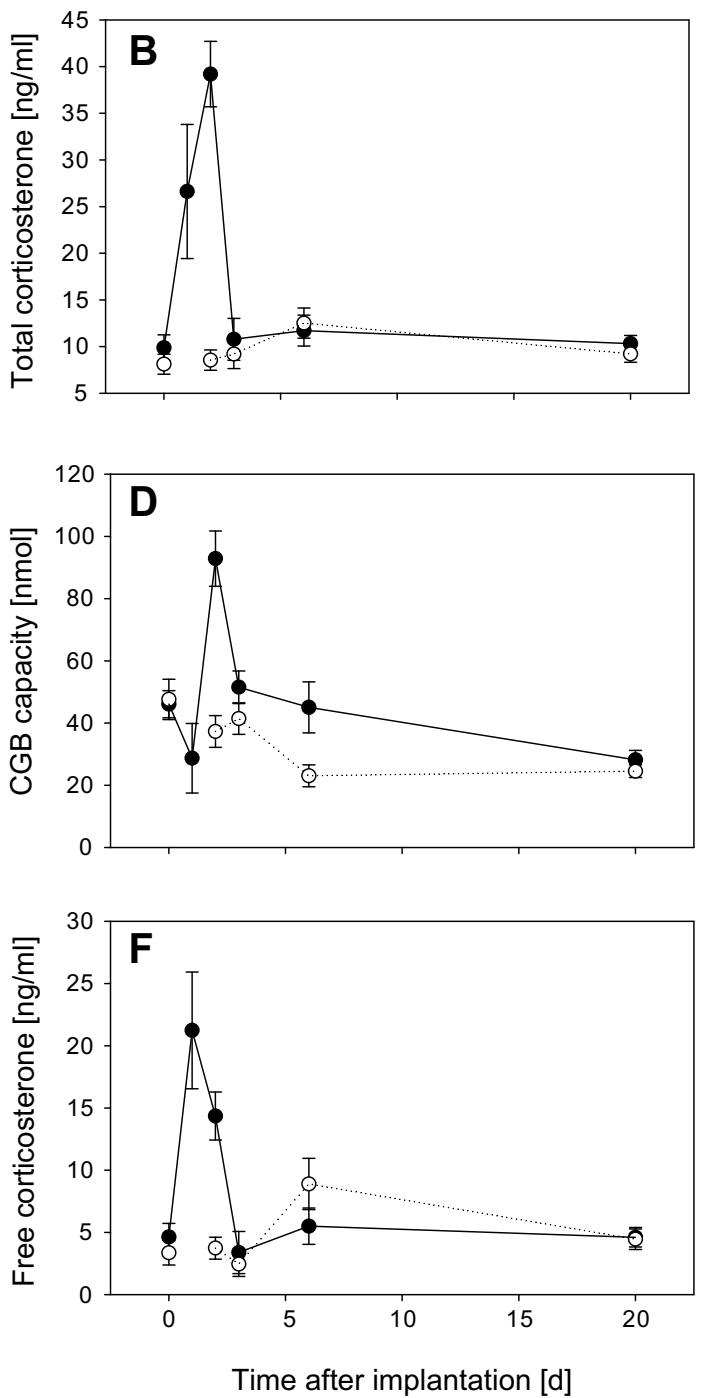

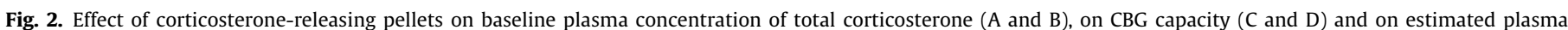

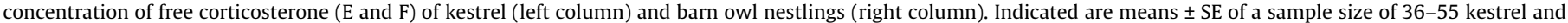

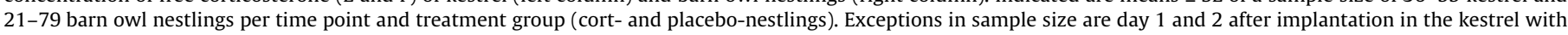

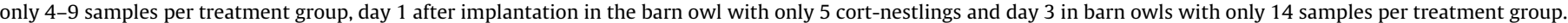
The pellet was implanted on day 0 , which corresponded to an age of 13 days in kestrels and 27 days in barn owls.

tlings (Fig. 3A and B). However, baseline corticosterone levels were not elevated to a constant level, but peaked 1-2 days after implantation, dropped to a lower level 2 days after implantation (in the kestrel) and had almost reached placebo-levels 3 days after implantation. Thus, with these pellets, corticosterone levels were elevated for a shorter period than the 7 days indicated by the provider and similar findings were obtained in house sparrows (C.W.B. unpublished data). There are two possible explanations. First, the corticosterone contained in the pellet may have been released very fast, resulting in peak-like levels higher than intended. It is possible, that the pellet matrix is metabolized faster and corticosterone released in a shorter time period in birds than in mammals, for which the pellet was designed originally. A second explanation is that through the internal negative feedback the release of endoge- 


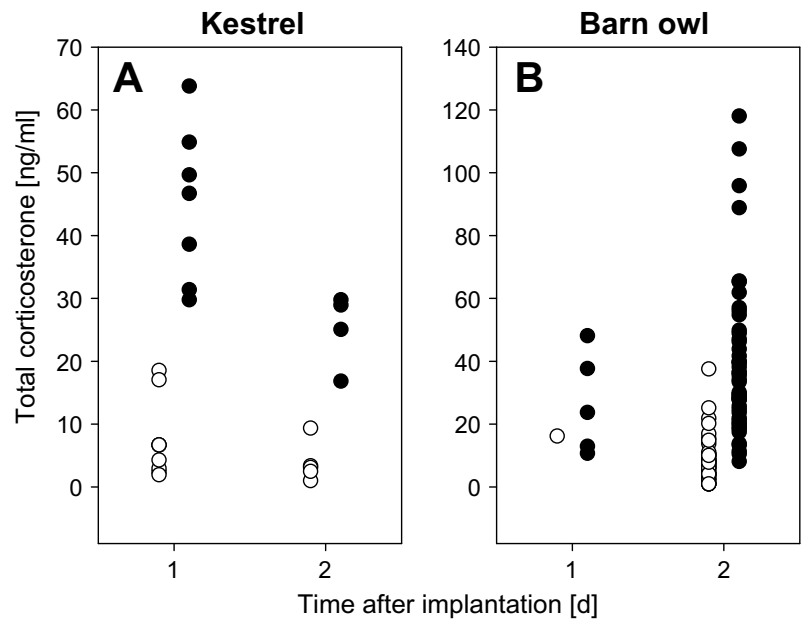

Fig. 3. Individual variation of baseline circulating total corticosterone levels during the two days after pellet implantation in (A) kestrel and (B) barn owl nestlings. All data points are from different individuals (filled symbols: Corticosterone, empty symbols: Placebo).

nous corticosterone was strongly reduced until the pellet was metabolized. The finding that the glucocorticoid response to handling was strongly reduced up to 8 days after implantation may suggest that the negative feedback mechanism was indeed in oper- ation (see below). Future studies, using pellets with labeled corticosterone, are needed to determine the relative amounts of endogenous and exogenous corticosterone in the blood and, thus, the importance of the internal negative feedback mechanism to regulate plasma levels of corticosterone after external administration.

In a study implanting silastic tubes filled with corticosterone in starlings Sturnus vulgaris and black-legged kittiwakes Rissa tridactyla, plasma corticosterone levels also had decreased to near placebo-levels 3 or 5 days after implantation (Romero et al., 2005; Angelier et al., 2007), also indicating a potential negative feedback regulation reducing the adrenocortical corticosterone release after some days. Studies using other techniques to administer corticosterone unfortunately do not present the time course of circulating corticosterone after administration. Usually, the resulting circulating corticosterone levels were measured only once at varying time points after the beginning of administration, which prevents the comparison of the time course of corticosterone levels between different methods.

The variation between individuals in baseline corticosterone levels 1-2 days after implantation was quite large (Fig. 3). Similar data from published studies using other ways of administering corticosterone are not known to us. It is well established that baseline and handling-induced corticosterone levels (not induced by implants) vary considerably between individuals (e.g. Cockrem and Silverin 2002), hence, there is considerable individual variation in the functioning of the HPA axis that may also hold when

Table 3

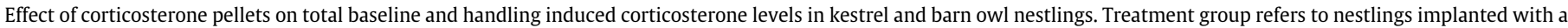

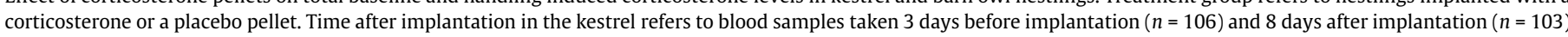

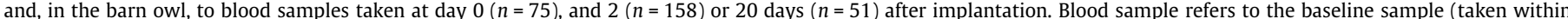

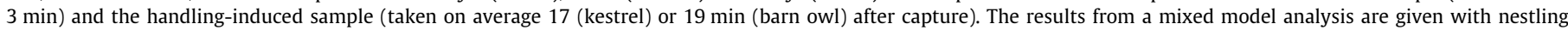
identity nested in broods as random factors.

\begin{tabular}{|c|c|c|c|c|c|c|}
\hline & \multicolumn{6}{|c|}{ Total corticosterone } \\
\hline & \multicolumn{3}{|l|}{ Kestrel } & \multicolumn{3}{|l|}{ Barn owl } \\
\hline & df & F statistic & $\mathrm{F} \mathrm{pr}$ & df & F statistic & $\mathrm{F} \mathrm{pr}$ \\
\hline Time after implantation & $1,321.7$ & 7.50 & 0.007 & $2,430.2$ & 8.71 & $<0.001$ \\
\hline Blood sample & $1,313.9$ & 155.47 & $<0.001$ & $1,395.1$ & 367.96 & $<0.001$ \\
\hline Treatment group & $1,82.6$ & 18.32 & $<0.001$ & $1,122.9$ & 3.45 & 0.066 \\
\hline Time after implantation $\times$ Blood sample & $1,313.9$ & 9.67 & 0.002 & $2,396.1$ & 23.05 & $<0.001$ \\
\hline Time after implantation $\times$ Treatment group & $1,322.9$ & 39.94 & $<0.001$ & $2,476.5$ & 1.15 & 0.319 \\
\hline Blood sample $\times$ Treatment group & $1,313.9$ & 10.75 & 0.001 & $1,395.2$ & 20.90 & $<0.001$ \\
\hline Time after implantation $\times$ Blood sample $\times$ Treatment group & $1,314.0$ & 29.15 & $<0.001$ & $2,396.2$ & 8.25 & $<0.001$ \\
\hline
\end{tabular}
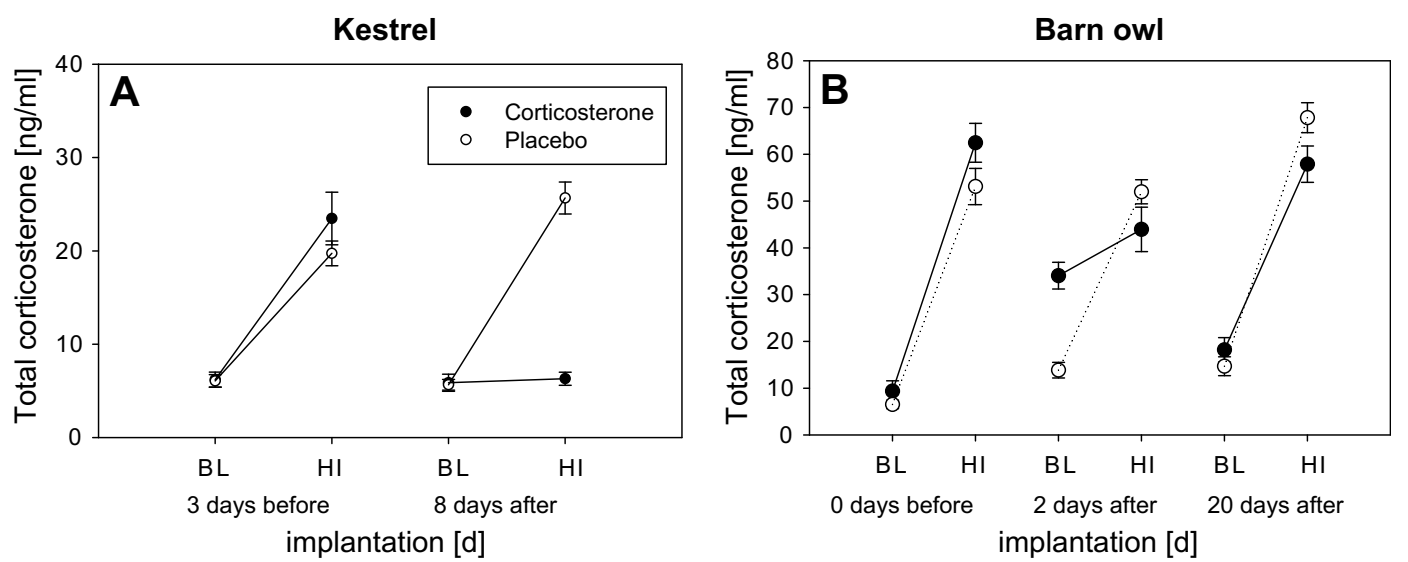

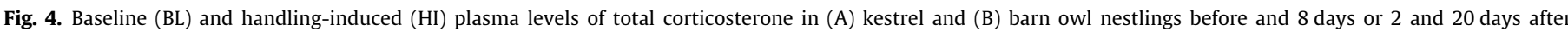

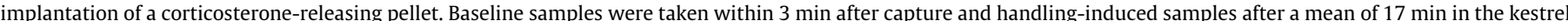

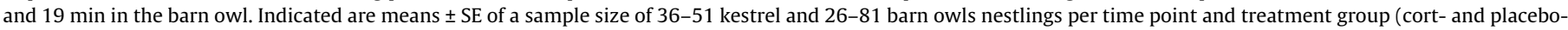
nestlings). The pellet was implanted on day 0 , which corresponded to an age of 13 days in kestrels and about 27 days in barn owls. 
administering corticosterone. We found that individual variation in corticosterone levels after implantation in barn owls depends on environmental conditions (Almasi et al., unpublished data) and are correlated with a genetically determined color trait (Almasi et al., unpublished data). Hence, the ability to buffer administered corticosterone seems to be an interesting individual trait in itself.

\subsection{Effect of corticosterone pellets on CBG capacity and free baseline corticosterone levels}

Concurrent to the increased corticosterone levels, the pellets also increased CBG capacity in barn owl nestlings and tended to increase CBG capacity (small sample size) in kestrel nestlings. This corresponds to increased plasma CBG in house sparrows Passer domesticus and serum CBG in mouse pups after glucocorticoid administration (Zhao et al., 1997; Breuner et al., 2003b), but is in contrast to a decrease in CBG production and secretion in rats after glucocorticoid administration (Feldman et al., 1979). It is possible, that the increase in CBG capacity serves to protect partly from the deleterious interferences of high free corticosterone levels with postnatal morphological and cognitive development (Kitaysky et al., 2003, Müller et al., unpublished data). However, the course of free baseline corticosterone correlated strongly with total baseline corticosterone levels, and the peak in total corticosterone concentration after pellet implantation was buffered only to a small degree by the simultaneously elevated CBG capacity. We did not find any impact of the corticosterone pellets on CBG capacity after the peak of circulating baseline corticosterone.

\subsection{Effect of corticosterone pellets on the adrenocortical response to handling}

There was only a small adrenocortical response to handling two days after corticosterone pellet implantation in barn owl nestlings and virtually no adrenocortical response to handling 8 days after corticosterone pellet implantation in kestrel nestlings. This can be explained by the negative feedback mechanism controlling circulating corticosterone levels (e.g. Keller-Wood and Dallman, 1984; McEwen et al., 1986). The negative feedback can occur as a rate-sensitive fast feedback and a level-sensitive delayed feedback (Dallman and Yates, 1969). The delayed feedback begins approximately 30 min following glucocorticoid elevation and extends for days. The duration of the subsequent inhibition of the axis depends on the absolute concentration of the steroids, thus may extend beyond the period of hormone administration (e.g. Abe and Critchlow, 1980; Sapolsky et al., 1986).

An attenuated adrenocortical response to an acute stressor, as we observed 2 days after implantation in barn owls, occurs when the animal is already under chronic stress as provoked here by corticosterone administration. As a result of a rate-sensitive fast feedback the hypothamalo-pituitary-adrenal axis shuts down and is not capable of mounting a response to an acute stress (Romero, 2004). One example in free-living birds is the absence of any further corticosterone elevation to capture and handling in a seabird, when a severe storm had already substantially increased circulating corticosterone (Smith et al., 1994). The absence of an adrenocortical response to handling in kestrel nestlings eight days after corticosterone pellet implantation (when baseline total corticosterone levels were not elevated anymore) may be explained by an extension of a level-sensitive delayed feedback. Alternatively, it may be explained by the continuous release of corticosterone from the pellet provoking the shut-down of the hypothamalo-pituitaryadrenal axis, as mentioned above.

Twenty days after corticosterone pellet implantation, the HPA axis seemed to have recovered from the corticosterone manipula- tion. As a response to handling barn owl cort-nestlings showed a similar increase in circulating total corticosterone levels as placebo-nestlings.

\section{Conclusions}

Self-degradable pellets are an efficient tool to artificially elevate corticosterone levels with one intervention. The dose and duration-dependent effects of corticosterone require the monitoring of the resulting circulating corticosterone levels. It is to be expected, that the release from pellets, and possibly from other implants, is not constant over time or that a delayed feedback sets in after a few days which both result in different plasma levels, and thus possibly different effects, of corticosterone at different stages during the experiment. The plasma levels resulting from corticosterone administration can be monitored in a subgroup within the experiment, in an additional group of implanted control animals or in a preliminary study. However, since the HPA axis can vary with season, life history stage, environmental conditions, age and sex (e.g. Wingfield et al., 1994; Kitaysky et al., 1999; Romero, 2002; O'Reilly and Wingfield, 2003; Love and Williams, 2008), any additional group should closely match the experimental groups and live under similar conditions and at the same time or season. Indeed, there is individual variation in corticosterone levels as a response to pellet implantation depending on baseline levels (own unpublished data) and nutritional conditions (Almasi et al., unpublished data).

When using self-degradable corticosterone pellets, no alcohol or similar solvent should be used during implantation, because even if seemingly evaporated, alcohol can strongly increase the release of corticosterone from the pellet. We also recommend to carefully determine the dosage of the pellet in test birds to avoid pharmacological levels during the first days after implantation.

The interactions of exogenous corticosterone with the negative feedback mechanism and the response to an acute stressor have to be taken into account in future studies. An increase in CBG capacity provoked by corticosterone administration results in an attenuated increase in circulating free baseline corticosterone levels compared with total levels, which may modify the effects of corticosterone on the animal.

\section{Acknowledgements}

We thank Ueli Schaffner, Renate Wullschleger, Martin Fischer, Martin Gassner, Linda Bischoff, Christophe Bonetti, Annette Sauter, Jodok Guntern, Matthias Ernst, Lena Senn, Andreas Rieser, Sonja Braaker, Annick Morgenthaler, Ester Pellegrini, Juliette Jullierat, Silvan Rüttimann, Pascal König, Martin Amrein, Silvain Antoniazza, Deborah Ramseier, Silvan Bissegger and Kim Stier for their help in the field. Marc Kéry gave valuable statistical advice. Financial support for this study was provided by the Swiss National Science Foundation Grant 3100A0-104134 to L.J and S.J-E and PPOOAO102913 to A.R.

\section{References}

Abe, K., Critchlow, V., 1980. Delayed feedback inhibition of stress-induced activation of pituitary-adrenal function: effects of varying dose, rate and duration of corticosterone administration and of telencephalon removal. Neuroendocrinology 31, 349-354.

Angelier, F. Clément-Chastel, C., Gabrielsen, G.W. Chastel, O, 2007 Corticosterone and time-activity budget: an experiment with black-legged kittiwakes. Hormones and Behavior 52, 482-491.

Barsano, C.P., Baumann, G., 1989. Simple algebraic and graphic methods for the apportionment of hormone (and receptor) into bound and free fractions in binding equilibria; or how to calculate bound and free hormone? Endocrinology 124, 1101-1106. 
Bonier, F., Martin, P.R., Wingfield, J.C., 2007. Maternal corticosteroids influence primary offspring sex ratio in a free-ranging passerine bird. Behavioral Ecology 18, 1045-1050.

Bourgeon, S., Raclot, T., 2006. Corticosterone selectively decreases humoral immunity in female eiders during incubation. Journal of Experimental Biology 209, 4957-4965.

Breuner, C.W., Greenberg, A.L., Wingfield, J.C., 1998. Noninvasive corticosterone treatment rapidly increases activity in gambel's white-crowned sparrows (Zonotrichia leucophrys gambelii). General and Comparative Endocrinology $111,386-394$.

Breuner, C.W., Orchinik, M., Hahn, T.P., Meddle, S.L., Moore, I.T., Owen-Ashley, N.T., Sperry, T.S., Wingfield, J.C., 2003a. Differential mechanisms for regulation of the stress response across latitudinal gradients. American Journal of Physiology 285, R594-R600.

Breuner, C.W., Wada, H., Shyu, J., Love, O.P., 2003b. Corticosteroid binding globulin capacity responds to chronic hormone treatment but not acute stressors. Integrative and comparative biology 46, 1013.

Cockrem, J.F., Silverin, B., 2002. Variation within and betweeen birds in corticosterone responses of great tits (parus major). General and comparative Endocrinology 125, 197-206.

Dallman, M.F., Yates, F., 1969. Dynamic asymmetries in the corticoid feedback path and distribution-metabolism-binding elements of the adrenocortical system. Annals of the New York Academy of Sciences 156, 696-721.

Donker, R.A., Beuving, G., 1989. Effect of corticosterone infusion on plasma corticosterone concentration, antibody production, circulating leukocytes and growth in chicken lines selected for humoral immune responsiveness. British Poultry Science 30, 361-370.

Feldman, D., Mondon, C.E., Horner, J.A., Weiser, J.N., 1979. Glucocorticoid and estrogen regulation of corticosteroid-binding globulin production by rat liver. American Journal of Physiology-Endocrinology and Metabolism 237, E493E499.

French, S.S., McLemore, R., Vernon, B., Johnston, G.I.H., Moore, M.C., 2007. Corticosterone modulation of reproductive and immune systems trade-offs in female tree lizards: long-term corticosterone manipulations via injectable gelling material. Journal of Experimental Biology 210, 2859-2865.

Hiebert, S.M., Ramenofsky, M., Salvante, K., Wingfield, J.C., Gass, C.L., 2000. Noninvasive methods for measuring and manipulating corticosterone in hummingbirds. General and Comparative Endocrinology 120, 235-247.

Horton, B.M., Long, J.A., Holberton, R.L., 2007. Intraperitoneal delivery of exogenous corticosterone via osmotic pump in a passerine bird. General and Comparative Endocrinology 152, 8-13.

Hull, K.L., Cockrem, J.F., Bridges, J.P., Candy, E.J., Davidson, C.M., 2007. Effects of corticosterone treatment on growth, development, and the corticosterone response to handling in young Japanese quail (Coturnix coturnix japonica). Comparative Biochemistry and Physiology-Part A: Molecular \& Integrative Physiology 148, 531-543.

Keller-Wood, M.E., Dallman, M.F., 1984. Corticosteroid inhibition of ACTH secretion. Endocrine Reviews 5, 1-24.

Kitaysky, A.S., Kitaiskaia, E.V., Piatt, J.F., Wingfield, J.C., 2003. Benefits and costs of increased levels of corticosterone in seabird chicks. Hormones and Behavior 43, 140-149.

Kitaysky, A.S., Wingfield, J.C., Piatt, J.F., 1999. Dynamics of food availability, body condition and physiological stress response in breeding black-legged kittiwakes. Functional Ecology 13, 577-584.
Kitaysky, A.S., Wingfield, J.C., Piatt, J.F., 2001. Corticosterone facilitates begging and affects resource allocation in the black-legged kittiwake. Behavioral Ecology 12, 619-625.

Loiseau, C., Sorci, G., Dano, S., Chastel, O., 2008. Effects of experimental increase of corticosterone levels on begging behavior, immunity and parental provisioning rate in house sparrows. General and Comparative Endocrinology 155, 101-108.

Love, O.P., Williams, T.D., 2008. Plasticity in the adrenocortical response of a freeliving vertebrate: The role of pre- and post-natal developmental stress. Hormones and Behavior 54, 496-505.

McEwen, B.S., de Kloet, E.R., Rostene, W., 1986. Adrenal steroid receptors and actions in the nervous system. Physiological Reviews 66, 1121-1188.

Munro, C., Stabenfeldt, G., 1984. Development of a microtitre plate enzyme immunoassay for the determination of progesterone. Journal of Endocrinology 101, 41-49.

Munro, C.J., Lasley, B.L., 1988. Non-radiometric methods for immunoassay of steroid hormones. In: Albertson, B.D., Haseltine, F.P. (Eds.), Non-radiometric Assays: Technology and Application in Polypeptide and Steroid Hormone Detection. Alan R. Liss, Inc., cop., New York, pp. 289-329.

O'Reilly, K.M., Wingfield, J.C., 2003. Seasonal, age, and sex differences in weight, fat reserves, and plasma corticosterone in Western Sandpipers. Condor 105, 13-26.

Pravosudov, V.V., 2003. Long-term moderate elevation of corticosterone facilitates avian food-caching behaviour and enhances spatial memory. Proceedings of the Royal Society of London-Series B: Biological Sciences 270, 2599-2604.

Remage-Healey, L., Romero, L.M., 2002. Corticosterone and insulin interact to regulate plasma glucose but not lipid concentrations in molting starlings. General and Comparative Endocrinology 129, 88-94.

Romero, L.M., 2002. Seasonal changes in plasma glucocorticoid concentrations in free-living vertebrates. General and Comparative Endocrinology 128, 1-24.

Romero, L.M., 2004. Physiological stress in ecology: lessons from biomedical research. Trends in Ecology and Evolution 19, 249-255.

Romero, L.M., Strochlic, D., Wingfield, J.C., 2005. Corticosterone inhibits feather growth: potential mechanism explaining seasonal down regulation of corticosterone during molt. Comparative Biochemistry and Physiology - Part A: Molecular and Integrative Physiology 142, 65-73.

Sapolsky, R.M., Krey, L.C., McEwen, B.S., 1986. The adrenocortical axis in the aged rat: Impaired sensitivity to both fast and delayed feedback inhibition. Neurobiology of Aging 7, 331-335.

Silverin, B., 1986. Corticosterone-binding proteins and behavioral effects of high plasma levels of corticosterone during the breeding period. General and Comparative Endocrinology 64, 67-74.

Smith, G.T., Wingfield, J.C., Veit, R.C., 1994. Adrenocortical response to stress in the common diving petrel, Pelecanoides urinatrix. Physiological Zoology 67, 526537.

Sokal, R.R., Rohlf, F.J., 2000. Biometry. W. H. Freeman and company, New York.

Wingfield, J.C., Deviche, P., Sharbaugh, S., Astheimer, L.B., Holberton, R., Suydam, R., Hunt, K., 1994. Seasonal changes of the adrenocortical responses to stress in redpolls, Acanthis flammea, in Alaska. Journal of Experimental Zoology 270, 372-380.

Wingfield, J.C., Silverin, B., 1986. Effects of corticosterone on territorial behavior of free-living male song-sparrows, Melospiza melodia. Hormones and Behavior 20, 405-417.

Zhao, X.F., Scrocchi, L.A., Hammond, G.L., 1997. Glucocorticoids induce corticosteroid-binding globulin biosynthesis by immature mouse liver and kidney. The Journal of Steroid Biochemistry and Molecular Biology 60, 163-169. 